\title{
Cris et gestes pour un acte de parole Le Coup de l'étrier de Claude Pierre
}

\author{
Jacqueline Beaugé-Rosier
}

Lobjet visé dans cet article est un micro-univers implicite aux textes poétiques, Le coup de l'étrier de Claude Pierre. Nous laisserons entrevoir que cet acte langagier fait de cris et de gestes est particulier en tant qu'un produit manifeste de l'aire socio-culturelle haïtienne de l'exil.

En fait nous utiliserons un langage déjà construit, l'histoire d'un poète lancée à la gestuelle du coup de l'étrier. Cette histoire, née des motivations qui s'intègrent à l'oeuvre elle-même, est susceptible de rendre compte de son contenu.

Dans cette perspective nous aurons en mémoire que la mise en circulation de ces textes poétiques témoigne de l'universalité du sens des propos de l'écrivain, Claude Pierre, assis à la barre du souvenir et de l'aube naissante.

Tout d'abord le titre manifeste de l'oeuvre est un médiateur issu des mêmes sources que le double geste. Dans sa complexité morphologique comme dans le figement de ses sens mythiques, ce médiateur oriente un voyageur en instance de départ vers les "temps de la commune humanité" (Célébration, p. 17).

En observant l'association des lexèmes, boire le coup de l'étrier, c'est-à-dire le dernier verre avant de partir, on se trouve en présence de deux actes contigus.

A priori, la levée des verres, c'est stricto sensu, et littéralement le rituel d'un hommage prolongé dans un continuum. Ce pôle ne peut être vu que dans l'élévation des mains qui marque un faire humain: ascension de l'esprit vers une vision plus ouverte de la vie constante.

En second lieu, le départ touche profondément le mobile et le sonore des pas à l'affût de cette ascension. Entre le faisceau des mains et l'espace qui s'ouvre à la découverte, la vie constante cristallise l'éloignement et transpose l'éventuelle absence dans un ailleurs présent. 
On peut alors rendre compte de ce que ce dëpart suggère dans la mesure où le geste posé correspond à un désir souhaitable de l'homme qui s'en va à la quête de s'abîmer aux sources d'une "réminiscence baignée dans une neuve atmosphère." ${ }^{1}$

N'étant plus une solution individuelle et angoissante, le jeu de mots cesse d'être externe à l'histoire. Il opère la mise en scène d'un acte conscient/inconscient qui a pour base le langage et le monde et qui déplace les sens.

Trinquer le verre de l'adieu avant le départ inscrit une gestuelle qui porte, d'une part, les cris et les coeurs réunis à la table du partage. D'autre part, ce signe de l'union du voyageur omniscient, tiraillé entre le monde d'ici et d'ailleurs, n'est pas un pur hasard. La présence unifiante se veut refuge, durée, lieu de rencontre, hors la contrainte et la mélancolie des désirs, hors l'amertume des sympathies interrompues et parce que le Désir élargi englobe les événements passés-présents et l'inconnu d'un avenir, éléments indissociables de la quête et du courage. Une présence qui nous met en contact d'un temps réel suspendu dans la vide des choses et les cris du vent.

Et c'est évidemment la terre de l'écrivain qui doit mener à la voie. Cette terre agit et ne se retire jamais. La mystique du geste se place au coeur de l'imaginaire

"point d'eau où laver ses ulcères."

Et ce qui submerge c'est la vérité du texte conjointe à l'exactitude des informations. C'est la mise en discours qui accorde une présence au dialogue.

Compte tenu de son intuition personnelle et de sa vision du monde, chacun s'avère libre d'approcher "la source où désaltérer sa course." Car au-delà de la matière-espace-temps, la mémoire est toujours présente.

Nouvelle expérience conjointe à la vie médium, "le coup de l'étrier" nous livre une fois de plus des symboles tranchés dans leur substance première. En direct, la parole de l'écrivain, présente, actualisée, sous-jacente de l'imaginaire, autrement puissante que l'expérience et l'identifiable des choses évoquées. 
Du début à la fin de l'oeuvre, cette parole demeure absolue, identifiable au poète. Car, selon toute évidence, "ce ne sont pas les mots où les phrases, mais au contraire les hommes qui signifient au moyen des mots et de la phrase. Le signifié est fonction de l'usage, mais d'un usage socialement réglé et coordonné." ${ }^{2}$

A titre d'exemple, la tonalité des sèmes du "tire-bouchon" reproduit au niveau du symbole un langage immanent issu d'un sixième sens. Il s'y mêle le fondement d'un pouvoir naturel et d'une espérance profonde greffés à la préoccupation du poète: «aérer la voie pour demain."

Comme le "lait qui marronne la mort et qui apaise le temps," la réalité douloureuse de la terre natale, nouvellement libérée et démunie, vit au coeur de l'oeuvre. Tous les motifs évoqués dans l'espace cyclique s'apparentent à un savoir mystique transmis aux "pieds-bois," dont l'arbre centenaire témoin, par les drisses du vent nomade. Ce frère hybride d'un personnage mythique, le bouche en bouche, atteint dans son essence même la sensibilité populaire de l'Haïti d'aujourd'hui. A cette fin, la remontée à la source privilégie le visage humain d'une relation possible qui constitue le point de départ de l'harmonie créatrice. Pour le poète-Autrui-le Monde, l'espoir est au bout du chemin.

Nul doute que dès les premiers cris, l'oeuvre et le geste affichent une intention pédagogique "élever au plan d'âme le temps de la commune humanité (Célébration, p. 17). D'où l'entrée en relation d'après les rites qui nécessite un apprivoisement progressif."

Dresser des bacs en offrande à la mer et aux ètoiles pour ménager la susceptibilité des dieux et acheter leur assentiment.

(Tire-bouchon, p. 18)

Habituellement l'apprentissage peut susciter diffërents intérêts: épanouissement, matière à conflit, aliénation. Et si nous regardons les intervenants sociaux nous aboutissons à une sorte de transition entre notre Moi et le Moi de l'Autre. Le secret à percer incite au raisonnement. L'oralité du témoignage vécu et repensé s'impose de nouveau à la réflexion à cause de la force interne du dire qui crie "non au naufrage," par le fait même que le dire est donné à voir 
l'essentiel, "la naissance d'un jour où la parole sera signifiance de pollen" (D'encens et de soufre, chant X, p. 53).

A ce point de vue, la relation proposée par l'auteur aboutit à une synthèse créatrice de sens qui enrichit le monde.

En effet voici que mobilisée par une poussée grandiose et puissante, l'urgence de la quête signifiante survole "le grabat des indifférences." Le silence d'un acte langagier se fait chant d'un bout à l'autre de la terre. Et ce chant s'adresse non seulement à celui qui regarde, mais encore et surtout à qui prend en compte et intègre la mémoire, c'est-à-dire l'espace où l'action évolue.

Que dire de la mise en forme de l'oeuvre sinon qu'elle s'expose elle-mème à la vue en tant que détermination d'une vision à départager à l'appui de motifs et d'exemples tirés de la vie douceamère.

Outre ces lignes de forces qui s'imposent à notre compréhension, chaque poème, chaque séquence reviennent par ricochets nous atteindre sous forme de prises en charge. Si bien qu'un coup d'oeil d'ensemble peut nous restituer le micro-univers poétique ainsi révélé à notre entendement.

Équilibre et harmonie maintiennent l'ossature de l'oeuvre. Le jeu de l'expression assure la valeur des jeux sonores. Peinture éclatée, virile, pleine de force persuasive. Essentiellement la parole est le Domaine. Courbes du rythme. Verticalité des tableaux. Somme toute une sémiologie de discours revêtue de sa propre marque. Ici et là, le jet de cette parole souveraine privilégie les courants de la pensée. Et dans la plurivalence du dire, l'habitacle marin, aphrodisiaque, fraternel, fratricide, semble superposer des miroirs qui subjuguent la mémoire. Comme l'oeuvre elle-même qui vit et qui regarde, cette mer multiple et seule n'a pas de choix. Elle est vrille aux entrailles de ceux dont elle est source et dérive. Tout le dit se déverse en crues ardentes, en espaces pétillants d'ironie et d'amertume, de joies sous-jacentes.

Oeuvre d'architecture pluridimensionnelle d'une parole non robotisée, sévère parfois déchirante, "le coup de l'étrier" se fait fabulation d'un faire et convie le lecteur à explorer le bon et le mauvais, ce locuteur naturel de la vérité et du mensonge, ce porteparole de la peur fauve de l'être et de sa rédemption. 
Le Coup de l'étrier

67

Compte tenu de la graduation des textes poétiques, nous constatons que le bagage émotionnel dont est chargée l'oeuvre nous associe à la connaissance en nous révélant quelque peu les données de l'histoire. Histoire que nous nous proposons de présenter sous deux plans distincts: l'axe de la dimension passionnelle et poétique; l'axe de l'expérience.

Dimension passionnelle et poétique

Le coup de l'étrier constitue, à notre point de vue, le poète de retour au pays natal, le poète de l'exil qui s'interroge sur un avenir incertain. Un personnage suivi par le gars des vents en maraude, le nomade, le marron des bois, l'homme perpétuellement sur le qui-vive, figure bivalente d'un personnage omniprésent dans la mythologie haïtienne: le vent grand diseur.

Nous imaginons aisément qu'une écoute attentive d'un tel cheminement peut accoutumer le coeur en éveil à toucher du doigt les réalités physiques et mensongères si fortement dénoncées par le dire. Et quiconque voulant s'identifier à ce témoignage, si nous osons l'affirmer, répond de ce fait à l'appel fraternel du poète. Double perspective de "l'oracle qui ne ment jamais." Prétexte à croire le fait reconnaissable que toute vie possible se construit et s'organise dans une confrontation rigoureuse de l'instinct de vie par l'instinct de mort.

En quelque sorte vivre "à l'échelle d'enfer" c'est d'une part s'esquinter dans un corps à corps suicidaire, confronter des secousses inévitables, recevoir des chocs brutals, disparaitre ou ne plus exister, bref, c'est s'apprivoiser à la saisie d'un monde viscéral, stratifié, vrillé autant par la peur que le défi.

D'autre part, dans la mesure où l'individu lutte pour vivre, il s'avère impossible de refuser le combat. Et quelle que soit la forme de rencontre choisie, l'on peut s'attendre au meilleur et au pire. Faire preuve de courage et de patience à la manière d'un artisan conscient et honnête devient une attitude formelle, un enseignement à l'égard d'un présent immédiat et difficile: apprendre à espérer et à survivre.

A ce niveau l'écrivain ne se contente pas de dénoncer les agressions quotidiennes dont nous sommes victimes. Agressions de toutes sortes qui émoussent notre intelligence et nous obligent à forger une 
carapace protectrice sans aucun doute. "La rue encerclant son cou, les mains plantées d'araignées, de sauterelles, de limaces, l'âme à nu, ...," le poète fustige à coups de cravache le système ombilical, les stéréotypes infantiles, les préjugés tenaces, la délation, la mort lente au cachot, la mort dans la solitude et la défaite, qui ne s'adressent jamais au raisonnement mais plutôt brisent l'élan initial de l'Homme.

Parvenu à l'ėminence de l'hexagone logique, le récit- fabulation ne dévoile pas seulement la procession des objets-héros. La mise en scène se greffe au geste du poète en propulsant les "ahan, les oh là là inarticulés de l'intuition au-delà de l'éloge de la résistance."

Et cet écho sublime, qui sait, ne fait que traduire encore les obsessions et les tempêtes d'une âme en dérive.

En sens inverse, la synthèse évocatrice d'une future présence (arc-en-ciel de paix et d'amour) dote les marques du temps à renouveler d'un certain privilège. Une fois de plus la réalité des gestes s'inscrit dans la constellation du mythe de la Parole généreuse et ardente de l'écrivain.

Le pollen signifiant est, à ce titre, le signe visible de l'épanouissement du poète et du monde après l'épreuve. Mais comment y parvenir hors les limites de la vie constante?

Cette permanence de l'étendue circonscrit le dialogue-souvenir dans l'inexprimable de la surdité.

Et le drame quotidien prend alors un autre tournant. A force de crier dans le vide, l'homme perd la voix. Bien qu'il possède les outils, il ne peut aller au bout de laventure. Or il y a urgence. Il lui faut nécessairement retrouver sinon la parole du moins le cri.

Que faire devant un tel dilemme? Haranguer le vide? Aller vers celui qui dėtient le secret de la voie à suivre?

Face à cette alternative, l'Homme incapable de communiquer avec le monde s'enfonce dans le vent pour briser l'obstacle. Car il lui faut réapprendre à réinventer le songe entrevu en repartant à zéro.

De se reporter à la réminiscence des choses sous les auspices du vent met en relief l'étude d'une vérité à promouvoir selon une thérapeutique salvatrice, un paradoxe des valeurs sociales, culturelles, idéologiques, contestées jusqu'ici par le terrorisme pédagogique d'un héritage aliénant et dont les textes Mascarade, pour ne citer que ceux-ci nous en donnent certaines modalités. 
En moi rien il n'était rien donné de moi

d'aimer de chérir d'admirer

mes yeux sont taillés expressément pour

voir toute chose proprement mienne

laide hideuse horrible

on m'a nourri d'un pain de honte

désaltéré d'une eau boueuse puante

comme le ventre d'un charnier à zombis

(Mascarade, p. 76)

Si forte est la motivation que l'inattendu s'organise dans une effervescence de cris. Voici qu'entre le sommeil et le songe la sonorité du vent se change en plaintes usées, en sanglots cassés, en raideur, en gémissements, en souffrance inarticulée. Logique des faits d'où filtre la prédominance du vent criard et de ses effets multiples imposés à l'homme (déveine, malchance, exil, mort). Il s'agit précisément du sentiment d'infériorité ressurgissant à perte de vue dans la tranchée des actes, des comportements, des fuites désespérantes d'un peuple aux abois. Devenu vantard et fataliste d'occasion, pour masquer l'échec et l'insuccès notoires, l'homme de l'île mutilée semble s'abandonner, ad vitam aeternam, à un delirium tremens, insondable et effarant. Car en ce lieu-espace-matière-temps, la douleur et le ridicule mènent de front une lutte en cul-de-sac, sans espoir humain de survie.

Allusion à la mer multiple et secrète enfermée dans la corne du vent, une mer prise à ses propres ruses d'aphrodisiaque, une mer qui mue son visage en tempête, qui convoite, déroute le voyageur et tue.

Il n'est que de constater la densité de la transparence poétique, "l'océan gobe mes vieux os," pour comprendre et saisir l'à-propos d'une telle clairvoyance.

Pour ainsi dire le contexte réfère non seulement à une histoire initiale, préexistant au texte, mais aussi à celle de l'exil de l'hommeècrivain que son sixième sens mène à d'autres chemins.

Car le sonore des mots transmis au monde extérieur dépasse le mobile humain. Il s'impose par la violence des cris et pénètre au coeur de la signifiance. Équivalence des attitudes, des comporte- 
ments et des changements survenus au cours de l'évasion et qui peuvent configurer deux univers déchirants et tragiques.

Effectivement, entre la rage de l'homme muré dans sa solitude et la rage de la tempête se jouent des passions identiques: aboutissement à la destruction des choses par le vent tributaire de la voix; aboutissement à la destruction des êtres vivants par l'homme fratricide.

Dans cette sphère angoissante, le poète doté de paroles est devenu aphone sous l'emprise du vent. Nous sommes en présence d'une double aliènation figurative:

perte de la voix $=$ absence d'échange $=$ inhibition

de l'Homme;

destruction de vie $=$ mort lente. ${ }^{3}$

Cependant la rage du vent n'est palpable que dans ses effets extérieurs alors que la rage des hommes s'insère dans leurs attitudes et leurs comportements.

En opérant un sondage naturel, notre connaissance originelle de ces choses nous amène à déceler la différence qui s'impose au niveau des faires. Le faire humain, vérifiable par les apports de sens, surgit dans l'intériorité du discours.

Car l'écriture n'est pas accessoire. Elle est au coeur de l'entreprise littéraire. Entreprise qui n'est autre que l'exploitation par l'écrivain de son environnement, de l'immédiat qui atteint nécessairement le Moi et Autrui, le temps et les choses.

souviens-toi, dit le poète, tu peux tout dire l'expression est ton outil.

(Et le vent dans le vent, p. 28)

\section{Axe de l'expérience}

Il est facile de constater un fait évident. Dans ce micro-univers imaginaire, le vent porte le pays et ses enjeux, social, politique, humain. Un pays envoûté à qui le vent grand diseur veut restituer le pouvoir de la parole et le cri. Du moins ce désir en gestation veille au coeur du témoin sonore qui vacille dans une absurde course 
contre la montre et qui faute de parole non exprimée perd ses moyens.

Face à l'absence d'un procès vie/mort; vie promise/aliénation, le faire du vent devient dérisoire parce que sa "parole d’air est creuse et stérile."

Face aussi à la rupture de l'équilibre la vision fermée abstrait la vérité du procès,

fixée, figée, éteinte, la parole se recroqueville au creux d'elle-même.

On peut se représenter que le vent-frère-nomade du bouche en bouche est pris aussi dans le tourbillon des désespoirs de l'homme et qu'il se souvient du partage. De telle sorte que le vent restitue la voix à l'Homme qui chante la conscience de l'Avenir renaissant.
les grands courants embouteillés dans ma gorge exaspèrent l'archet des luettes grêles en plaintes usées en sanglots cassés.

Et que l'homme du songe devenu grand diseur du vent et porteparole de la connaissance assume la totalité du témoignage.

C'est dire que le climat de l'homme/écrivain et du poète songeur n'est pas serein. Aussi bien son cheminement n'est pas utopique quand il parle de l'homme et de ses signes comme d'une solitude ouverte. A l'infini, le non-être c'est l'exil et la mort de l'homme pensant, une mort dans la solitude et la défaite. C'est encore le non-paraître et l'absence d'un langage humain, avons-nous déjà dit. D'où l'impossible apprentissage et l'impossible enseignement "à l'orée d'un jour qui se fait un peu tirer les oreilles" et de la "mort embarrassée de nulle politesse" qui nous met perpétuellement en conflit.

Car dans la vie qui reste un instrument à réaccorder à la périphérie des gestes,

la leçon n'est pas apprise

l'homme est un enfer pour l'homme 
sa perversion sa dérision

sur la table de l'homme

(Règnes, p. 58)

Aussi le vraisemblable éclaire par touches révélatrices la fragilité du songe qui se barde pourtant de logique. C'est alors la résurgence de la quête de l'amitié née de la rutilance des hauts faits de l'histoire, et cependant hantée par leur dissemblance cauchemardesque. L'unité de la dimension poétique se resserre autour du fait-souvenir et du fait rappel, thèmes essentiels de l'alliance. Comme une conscience en éveil, les lampes de Clèment Magloire Saint-Aude dotées de "noms qui brillent au firmament de l'action ailleurs et là" dialoguent simultanément de "l'échelle d'enfer des héros poètes" à la dérive de l'écriture, "vent dans le vent," dont l'aboutissement mène aux portes sensibles de l'illogisme et de la mémoire. Idéologiquement le sens émerge en s'habillant d'événements transmis par le cri du poète. Autrement dit, "cet acte langagier fait irruption dans le discours tragique pour marquer l'impossibilité du langage articulé (cf l'exclamation) mais peut aussi représenter l'idéal d'une pensée pathétique réduite au plus extrême dénuement." ${ }^{4}$

Que s'est-il passé au-delà de cette rencontre? Quelle infinitude secrète peut nous révéler la vision du poète?

Au plus fort de notre interrogation la marche du "vent dans le vent" nous propose une réponse possible. Car nous apprenons que le poète décide de "rassembler ses zémès sourds, ses gris-gris, ses griots aphones, ses tabous inflexibles" pour une prise de conscience de plus en plus radicale. Car où peut-on retrouver "son élément vivifiant" ses racines et ses adhérences sinon au coeur même de son pays opprimé et désaxé.

D'autant plus que l'histoire de ce peuple qui multiplie les portraits de la vie et de la mort au gré de ce qui se voit et qui les transmet à perte de vue, dans la mascarade, la misère la plus acide, les atolls d'enfer, les noyades et les tueries barbares - n'évacue pas la patience du poète.

Et si au cours de l'aventure, le chaos et le désordre des angoisses et des tribulations campent l'itinéraire de palabres, de pouvoirs occultes, bref d'un merveilleux où se bousculent plusieurs avenues: lutte pour la liberté vraie, folie verbale, illogismes, paraboles, 
l'hypnose souhaitée et subie dans la traversée n'a d'autre alternative que d'investir l'histoire poétique de signes manifestes: courage, espoir, survivance.

Entre le vertige émergé des vases clos des tabous et le lieutémoin, le procès d'énonciation affirme la relation lecteur/poète.

Entre le poète qui nous convainc de sa privation et le désir d'être optimiste et bon prophète, le pouvoir de l'interprétation n'est plus un faire destiné à endormir un pays qui tourne à la moulinette du vide.

Le texte nous accorde que le poète est investi. Et puisque celui-ci a bonne mémoire, fervent et sage, il devient l'artisan de la parole.

\section{Progression de la quête}

Il est essentiel de souligner que les reprises plus ou moins variées, puisées à même les fondements anthropologiques montrent l'intensité du drame et l'intérêt de la démarche.

Ce manque, cette faim d'être et de vivre antérieurs au parler humain produit une valeur d'incantation propre au mythe du verbe. La situation du poète est telle que la transe se dote du pouvoir de substituer la parole matrice à l'obsession.

Aussi le conflit réel et présent se répète comme un geste cyclique de par l'évocation du monde imaginaire et des zones de production de symboles.

au ras de mes racines hybrides

des hommes écorchés vifs par le vol la rançon

au ras de mes racines hybrides

les hommes les os broyés rongés des rats

au ras de mes racines hybrides

des hommes mutilés-miraculés

victimes d'éternité d'une guerre

sourde anonyme et permanente 
au ras de mes racines hybrides

des hommes drogués à mort

de méringues truculentes

au ras de mes racines hybrides

des hommes dépouillés d'humanité

... raturés comme un texte à refaire ...

voilà au ras de mes racines hybrides

(Tire-bouchon, p. 21)

Ainsi prenant conscience de son investiture, le mythe de la parole parachève la réflexivité de la mémoire en accentuant le problème des masques de l'être et du non-être. Dans son trouble et ses contestations, le poète accélère cette conjonction de l'Absence et de la mort mutilée mais toujours présente, telle que nous les restituent ces séquences du "vent dans le vent."

la mort a déjà frappé

de toute façon la mort frappe

et frappera

la mort comme un taureau décapité

a défoncé l'infernale machine du temps.

A partir de ces éléments génériques vrillant l'ancrage d'une vie promise, le pouvoir s'étrangle dans l'oralité des offrandes rituelles, exaltantes d'encens et de soufre. C'est la mime intuitive du "Je" goguenard, douloureux, fragile. C'est la concrétisation des éléments hétérogènes qui vont fournir la base d'une définition spécifique de l'humanité agressée; définition à laquelle s'ajoutent l'ordre spatial et l'ordre sensoriel. De ces deux plans complémentaires nous ne retenons que la dêmarche.

A priori, la recommandation du poète:

Pour la célébration d'un temps longtemps

attendu

le temps de la commune humanité ...

voici ... 
crée instanténément un passé à l'histoire.

Dès lors nulle convention nul règne perceptible nulle altération ne restreint le non prévisible du chant. Le cri végétatif montant à l'affût des sens et du "sang énervé," se fait prophète du "Je" vigilant et identifiable

Je suis l'écarlate misère venue du midi des tropiques

Je suis votre mauvaise conscience

l'envers de votre aveugle confiance.

(La danse des illes, p. 85)

Constater l'identité des attributs du poète conduit à avancer qu'à partir de l'étape d'une conscience lucide et éclairée s'ouvre indéfiniment le champ de l'action. Aussi bien l'homme s'applique-t-il à construire un monde possible qui reformule les sens.

Ainsi la patience active du temps laborieux déchire les ganses de la mer, raccommode en fils d'araignée les blessures des raz-demarées et des nordés de pleine lune. Ainsi la tranquillité de l'enfance sauvage et aphone butine et cherche "au paradis et au midi des quatre jeudis" le don de la parole sensée. Ainsi les jeux frénétiques du "Je" omniscient s'évasent de la norme, se jouent des pièges, propulsent des climats hybrides qui s'autocorrigent pour mieux définir le procès d’une réalité urgente, le "Je" libre du songeur.

Car le poète qui n'est dans le geste de l'écriture ni situation ni personne mais présence semble combler le manque du début. Et dans sa course et sa quête de l'objet perdu, une vie harmonieuse d'entre les hommes de bonne conscience, le poète-songeur élabore les plans de la nouvelle naissance à l'inverse de l'espoir souhaitable et du rythme déchaîné de ses édens insulaires.

Dans ce contexte socio-culturel, la mesure du Temps ne peut être réduite à l'exactitude des sons langagiers ou à la perception des variables possibles si les constituants ne convergent au décodage du sens virtuel de l'oeuvre, "le tire-bouchon insulaire."

Et c'est pourquoi dans cet ordre d'idées l'oeuvre éclaire ellemême un chemin "à la bonne vôtre où l'on se salue à la sauvette" mais qui cependant nous ménage des espaces sereins. 
Ce rendez-vous auquel l'auteur nous convie fortement devient l'expression d'une liberté encore rongée par le couchemar, la terre de l'exil, et l'amitié dans l'exil, le chant du pays aimé et reconquis, la rédemption de la terre natale dans un monde reconstruit.

Et c'est à notre avis l'évocation d'une expérience humaine fondamentale, celle du destin, de la condition de l'homme, de la révolte et de la mort qui donne ouverture à la dignité retrouvée.

C'est une oeuvre qui a su rendre souverainement et par touches successives dans des textes riches et denses les cris de détresse d'un peuple, certes, mais également de protestation, de confiance, d'amour et d'espoir.

Et nous pouvons attester que l'homme-écrivain investi d'un personnage "nu et déserté de la vie" est lancé dans un présent en perpétuelle transition qui aliène et détruit son Etre pensant.

Il appert que Le coup de l'étrier de Claude Pierre chante la multiplicité d'une poésie de la logique, de la déraison et de la solitude.

D'une part, de par leur valeur didactique, les textes révèlent la démarche d'un personnage qui malgré sa vulnérabilité, donne un enseignement par le contraire d'un geste non aliénant. Car au plus fort de l'alienation physique et mentale, l'homme s'arme d'adresse, de ruse et de courage pour parvenir à l'échelle du jour. D'où le refus de l'absurde de l'être pensant en perpétuelle migration.

D'autre part, les valeurs anthropologiques (rites, magie, prières, religion, culture, race, coutumes) incitent le coeur et l'esprit à puiser l'écoute de sa vérité "aux abysses de la mémoire."

En conséquence, pour échapper à l'aliénation, pour ne plus commettre les erreurs du passê, l'homme-vigie devra, en toutes occasions, vérifier l'information reçue et en faire son double profond.

Ainsi se définit à notre entendement la leçon du poète et du songeur:

Écoute ne te laisse pas divertir ma parole est ton projet ton ouvrage ta création elle ne saurait sans ton support façonner sa destinée ma parole est toi tu la vivifies tu es l'oreille qui suppute d'un geste sûr sa chance 
dans la mer folle où tant de voix assassinent l'écho

Cette chance de te parler dans les mots que toi-même m'as appris m'enivre et je me nourris de cette parole pour que tu te souviennes.

Nous demeurons persuadés que le geste allié au cri demeure à l'égal du poète-homme-écrivain, authentique et sincère.

Car la poésie est pour l'écrivain. Claude Pierre, une occasion renouvelée d'exalter le courage d'un peuple qui a été mis à nu. Une raison valable d'espérer et de témoigner, malgré la solitude de l'acte, que la création poétique est une invitation à retrouver dans les sources la sagesse et la générositė d'une terre qui ne cesse de se chercher et de survivre.

\section{Notes}

'Stéphane Mallarmé, Crise des vers (Paris: Gallimard, Bibliothèque de la Pléiade), p. 368.

${ }^{2}$ Tullio de Mauro, Une introduction à la sémantique, (Paris: Payot, 1976), p. 196.

${ }^{3}$ André Niel, Dimension passionnelle et poétique, Analyse structurale des textes (Paris: ed. Mame, Coll. Jean-Pierre Delarge, 1973), p. 14.

André Niel parle de la relation moi-autrui-le monde, dans son ouvrage, La praxéologie de la pensée et de l'action.

Cette idée de structure de la relation-sujet-monde a été présentée par tous les psychologues, mais c'est l'idée moderne de "pattern de personnalité" et de "pattern de comportement" qui s'en approche le plus, un "pattern" est un modèle modelant, une forme constante de 
structuration active, in Roger Muchielli, Introduction à la Psychologie structurale (Paris: Dessart, 1966), p. 257.

${ }^{4}$ Jean Molino et Joeile Tamine, Introduction à l'analyse linguistique de la poésie (Paris: P.U.F., 1982), pp. 179-180

\section{Bibliographie}

Adam, J.M. et Goldenstein, J.P. "Les actes de parole, Linguistique et discours littéraire", in Searle, J.R. Les actes de langage. Paris: Herman, 1972.

Mallarmé, Stêphane. Crise de vers. Paris: Gallimard, Bibliothèque de la Pléiade.

Molino, Jean et Tamine, Joeile. Introduction à l'analyse linguistique de la poésie. Paris: P.U.F., Linguistique nouvelle, 1982.

Niel, André. L'analyse structurale des textes. Paris: ed. Mame, Coll. Jean-Pierre Delarge, 1973.

Mauro, Tullio de. Une introduction à la sémantique (Paris: Payot, 1976). 